\title{
Brauchen wir neue pneumologische Berufskrankheiten?
}

\author{
Do we Need Newly Defined Occupational Diseases of the Respiratory Tract?
}

Seit der letzten Novelle der Berufskrankheitenverordnung hat der ärztliche Sachverständigenbeirat beim Bundesarbeitsministerium, Sektion Berufskrankheiten, Empfehlungen für zwei neue Berufskrankheiten im Bereich der Atmungsorgane geschaffen:

- Lungenkrebs durch polyzyklische aromatische Kohlenwasserstoffe bei Nachweis der Einwirkung einer kumulativen Dosis von mindestens 100 Benzo(a)pyren-Jahren $\left[\left(\mu \mathrm{g} / \mathrm{m}^{3}\right)\right.$ Jahre] (noch nicht als neue BK umgesetzt) und

- Lungenkrebs durch die Einwirkung von kristallinem Siliziumdioxid $\left(\mathrm{SiO}_{2}\right)$ bei nachgewiesener Quarzstaublungenerkrankung (Silikose oder Silikotuberkulose) (BK 4112, neu).

Bei beiden Konstellationen stand man vor der Aufgabe, bei der häufigsten Krebserkrankung des Mannes und einer der häufigsten der Frau durch elaborierte, epidemiologisch-statistische Verfahren eine Subgruppe einzugrenzen, „die nach den Erkenntnissen der medizinischen Wissenschaft durch besondere Einwirkungen verursacht sind, denen bestimmte Personengruppen durch ihre versicherte Tätigkeit in erheblich höherem Grade als die übrige Bevölkerung ausgesetzt sind“, Definition der Berufskrankheit, §9 Abs. 1 SGB VII.

Was bedeutet das eigentlich? Es ist nicht gleichbedeutend mit dem Verdoppelungsrisiko (attributabler Anteil beruflicher Einflüsse an der Gesamtkausalität > 50\%), aber das Verdoppelungsrisiko ist ein gut handhabbarer Cut-off.

\section{Erstens: Lungenkrebs}

Es gibt einen gewissen logischen Mechanismus, nachdem ein $\mathrm{Ar}-$ beitsstoff, den die MAK- und BAT-Kommission der Deutschen Forschungsgemeinschaft als erwiesenermaßen humankanzerogen in die Gruppe K1 eingestuft hat, hinsichtlich seiner BK-Relevanz aufgearbeitet werden muss. Die Tabelle, die auch zwei K2-Stoffe beinhaltet, mag das veranschaulichen (Tab.1):

Tab. 1 Pulmonale Kanzerogene - präventive und kompensatorische Klassifikation

\begin{tabular}{|c|c|c|}
\hline Noxe & $\begin{array}{l}\text { Prävention } \\
\text { (MAK-Kategorie) }\end{array}$ & $\begin{array}{l}\text { Kompensation } \\
\text { BK-Nummer }\end{array}$ \\
\hline Asbest & K1 & BK 4104, 4105 \\
\hline Quarz & K1 & BK 4101, BK 4112 \\
\hline Ionisierende Strahlung & $\begin{array}{l}\text { (Strahlenschutz- } \\
\text { verord.) }\end{array}$ & BK 2402 \\
\hline Arsenverbindungen & K1 & BK 1108 \\
\hline Dichlordimethylether & K1 & BK 1310 \\
\hline Zinkchromat & K1 & BK 1103 \\
\hline Dichlordiethylsulfid (LOST) & K1 & BK 1311 \\
\hline Nickel, -verbindungen & K1 & BK 4109 \\
\hline $\begin{array}{l}\text { Pyrolyseprodukte aus org. } \\
\text { Material }\end{array}$ & K1 & $\begin{array}{l}\text { BK } 4110 \text {, Erweiterung } \\
\text { wurde empfohlen }\end{array}$ \\
\hline Passivrauchen am Arbeitsplatz & K1 & keine \\
\hline Dieselmotoremissionen & $\mathrm{K} 2$ & keine \\
\hline Beryllium, -verbindungen & $\mathrm{K} 2^{*}$ & (BK 1110) \\
\hline
\end{tabular}

* derzeit Überprüfung der krebserzeugenden Wirkung auf den Menschen 
Daran ändert auch die Tatsache nichts, dass die „Schwelle“ für Prävention (MAK-Werte, BAT-Werte, TRK-Werte) und Kompensation (Berufskrankheiten-rechtliche Relevanz) verschieden hoch sind - erstere niedriger, letztere höher. Passivrauch ist heute momentan das einzige erwiesene Humankanzerogen mit dem Zielorgan „Atemtrakt“, das von keiner Listen-BK begleitet wird. Mit anderen Worten: Wer als nichtrauchender Gastwirt oder Mitropa-Bedienung (das sind die vor Rauch meist kaum zu erkennenden Damen und Herren im so genannten Bistro zwischen 1. und 2. Klasse in der Bahn) an einem Lungenkrebs erkrankt, bekommt derzeit keine Kompensation aus der Unfallkasse. Ist das richtig oder falsch? Ich weiß es nicht, die wissenschaftliche Diskussion hierzu läuft gegenwärtig sehr intensiv.

\section{Zweitens: Obstruktive Atemwegserkrankungen}

Vordergründig ist hier regulatorisch alles in Ordnung: Für allergisch bedingte obstruktive Atemwegserkrankungen gibt es die BK 4301, für chemisch-irritativ oder toxisch bedingte die BK 4302. Pneumologen sprechen gerne vom „Berufsasthma“, welches unter den BK-Nrn. 4301 und 4302 anerkannt und entschädigt werden kann. Aber der Wortlaut der Berufskrankheitenverordnung kennt nur die „obstruktive Atemwegserkrankung“, nicht Asthma, Bronchitis oder Emphysem. Schaut man in die wissenschaftliche Begründung anlässlich der Schaffung der BKTatbestände 4301 und 4302, liest man, dass ausdrücklich nicht an eine Ausweitung des Krankheitsbildes „Asthma bronchiale“ gedacht war. Bei Licht betrachtet: Die vergleichsweise wenigen Fälle von COPD, die viele Pneumologen und Arbeitsmediziner unter der BK-Nr. 4302 zur Anerkennung vorschlagen, werden wohl streng genommen contra legem anerkannt und entschädigt. Schlimm? Nein, weil wissenschaftlich richtig. Eine Aktualisierung würde medizinisch Sinn machen, aber es besteht nur geringer Handlungsbedarf.

\section{Drittens: Chronische Bronchitis}

Es gibt die chronische Bronchitis ohne und mit Obstruktion. Letztere wird - und sei es contra legem -, solange es medizinisch-wissenschaftlich richtig ist, wie gesagt, ggf. unter der BKNr. 4302 von wachen Kollegen mit bedacht. Für erstere gibt es keine BK-Nummer. Schlimm? Ja, denn die chronische Bronchitis ist eine chronische, ernste, risikobehaftete, auch hier wieder einmal unterschätzte Krankheit. Wozu aber sollte man einen BKTatbestand schaffen, der kaum mit einer messbaren Minderung der Erwerbsfähigkeit einhergehen würde? Natürlich um der Prävention Willen! Kein Unfallversicherungsträger kann und darf Präventionsmaßnahmen nach §3 BKV leisten, wenn keine BK droht. Und wenn die chronische Bronchitis für Schweinehalter, der chronisch gegenüber anderweitigen organischen Stäuben ex- ponierten Personen und die chronische Bronchitis der Schweißer keinem BK-Tatbestand entspricht, kommen wir in puncto BKPrävention nicht weiter. Wir können nur 10-15 Jahre abwarten, bis die chronische Bronchitis mit einer Obstruktion einhergeht, dann winkt die BK 4302 auf hohem intellektuellen Niveau, aber zu spät. Hier sehe ich Handlungsbedarf.

\section{Viertens: Interstitielle Lungenerkrankungen}

Die Gruppe der Gießener Arbeitsmediziner um Woitowitz präsentiert in diesem Heft eine Fallserie von Erkrankungen an Lungenfibrose bei Schweißern - die Schriftleitung hat mir gestattet, die Tragik eines dieser Patienten - Siderofibrose, atypische Mykobakteriose, Lungentransplantation - kasuistisch auszuführen. Nun hat Mitleid nichts mit einer soliden Kausalitätsbeurteilung zu tun, Mitleid kann die Urteilskraft trüben. Was ist also mit der von mir oben im Abschnitt „Erstens: Lungenkrebs“ abzuleitenden Forderung, sich nur auf Odds Ratios und Konfidenzintervalle zu verlassen? Die Forderung ist hier plötzlich merkwürdig ungriffig - sie ist es bei nahezu allen seltenen Krankheiten. So berechtigt sie bei häufigen Krankheiten ist, so sehr geht sie bei seltenen Krankheiten an der Sache vorbei. Und die Expositionsbedingungen, unter denen die hier beschriebenen Lungenfibrosen entstanden sind, sind so extrem, dass es - gottlob - nur Kasuistiken gibt. In ganz Deutschland handelt es sich hierbei vielleicht um 30 bis 50 dieser Patienten, sicherlich nicht mehr als 100. Diese Fälle entziehen sich üblichen Statistiken, da sie - wiederum gottlob - weniger als $1 \%$ aller Schweißer ausmachen. Die Gruppentypik sehe ich als Webfehler des BK-Systems, denn einerseits sind Berufskrankheiten den Arbeitsunfällen seit Bismarcks Zeiten rechtlich gleichgestellt, andererseits wird für Berufskrankheiten eine Gruppentypik gefordert, für Arbeitsunfälle nicht: Es müssen eben nicht Serien von Industriearbeitern mit heißem Aluminium übergossen werden, oder bei Glatteis auf dem Weg zur Metallhütte in den Graben fahren, und es müssen nicht große Zahlen von Professoren auf studentischen Bananenschalen ausrutschen, damit die Einzelfälle als Arbeits- oder Wegeunfälle anerkannt werden können. Der Weg des Sonderentscheids in der ehemaligen DDR war für dergleichen Fälle nicht schlecht. Der Gesetzgeber hat hierzulande diese Ungleichbehandlung zwischen Arbeitsunfällen und Berufskrankheiten wohl gewollt, medizinisch-ethisch können wir sie nicht akzeptieren.

Zurück zur Lungenfibrose der Schweißer: Wenn neben den hier versagenden Odds Ratios und Konfidenzintervallen die international wissenschaftlich akzeptierten Kausalitätskriterien von Hill oder anderen Autoren angewandt werden, die nicht nur epidemiologische Evidenz gelten lassen, spricht m. E. alles dafür, diese Krankheit in die Liste der Berufskrankheiten aufzunehmen. 